\title{
CARACTERÍSTICAS SOCIOCULTURAIS E CLÍNICAS DE ADOLESCENTES COM DIABETES MELLITUS TIPO 1
}

\author{
Amanda Newle Sousa Silva ${ }^{1}$, Milena Siqueira Apolonio ${ }^{2}$, Caroline Magalhães de Alcantara ${ }^{2}$, Maria Veraci \\ Oliveira Queiroz ${ }^{3}$
}

\begin{abstract}
RESUMO: Objetivou-se descrever os aspectos socioculturais e clínicos de adolescentes com Diabetes Mellitus tipo 1 e relatar atividade educativa em grupo com base nas necessidades do público-alvo. Estudo quantitativo, com 38 adolescentes, ocorreu de janeiro a junho de 2015, em Fortaleza, Ceará, Brasil. Dados descritos com frequências percentuais. Das reflexões, obteve-se insight para atividade educativa, com cinco adolescentes escolhidos, intencionalmente. Maior parte residia em Fortaleza 20 (52,6\%), morava em casa própria 32 (84,2\%), com renda familiar de um a dois salários mínimos 35 (92,1\%). Os adolescentes pesquisados apresentaram peso adequado 20 (52,6\%), hiperglicemia em jejum 27 (71\%), utilizavam dois tipos de insulina $18(47,3 \%)$. Enfatizaram maiores dificuldades no controle glicêmico relacionado à alimentação. Realizou-se prática educativa em grupo sobre este obstáculo. Os sujeitos pesquisados detinham conhecimentos sobre a doença e os cuidados; as dificuldades na adesão ao tratamento envolviam aspectos socioculturais que poderiam ser negociados com estes e familiares.
\end{abstract}

DESCRITORES: Enfermagem; Diabetes mellitus tipo 1; Saúde do adolescente.

\section{SOCIOCULTURAL CHARACTERISTICS OF ADOLESCENTS WITH TYPE 1 DIABETES MELLITUS}

ABSTRACT: The present study aimed to describe the sociocultural and clinical aspects of adolescents with type 1 Diabetes Mellitus and report group educational activity based on the needs of the target audience. Quantitative study with 38 adolescents conducted from January to June 2015, in Fortaleza, Ceará, Brazil. Descriptive statistics was used. Insight was gained to educational activities, with five adolescents intentionally selected. Most of them lived in Fortaleza: 20(52.6\%), lived in their own houses: 32(84.2\%), family income of one to two minimum wages: 35(92.1\%). The participants had normal weight: $20(52.6 \%)$, fasting hyperglycemia: $27(71 \%)$, used two types of insulin: $18(47.3 \%)$. They reported higher difficulty in adhering to healthier eating habits to control blood glucose levels. A group educational activity focused on this obstacle was conducted. The subjects had knowledge about the disease and related care; the difficulties in treatment adherence involved sociocultural aspects that could be negotiated with the subjects and their families.

DESCRIPTORS: Nursing; type 1 Diabetes mellitus; Adolescent health.

\section{CARACTERÍSTICAS SOCIOCULTURALES Y CLÍNICAS DE ADOLESCENTES CON DIABETES MELLITUS TIPO 1}

RESUMEN: La finalidad del estudio fue describir aspectos socioculturales y clínicos de adolescentes con Diabetes Mellitus tipo 1, así como relatar actividad educativa en grupo, considerando las necesidades de ese público. Estudio cuantitativo, con 38 adolescentes, hecho de enero a junio de 2015, en Fortaleza, Ceará, Brasil. Los datos fueron descriptos con frecuencias percentuales. De las reflexiones, se obtuvo insight para actividad educativa, con cinco adolescentes seleccionados de modo intencional. De los participantes, 20 vivían en Fortaleza $(52,6 \%), 32$ vivían en su vivienda (84,2\%), 35 presentaban renta familiar de uno a dos sueldos mínimos (92,1\%). De los adolescentes investigados, 20 presentaron peso adecuado (52,6\%), 27, hiperglucemia en ayuno (71\%), 18 utilizaban dos tipos de insulina (47,3\%). Se constataron mayores dificultades en el control de glucemia referente a la alimentación. Hubo práctica educativa en grupo acerca de esa cuestión. Los sujetos investigados poseían conocimientos sobre la enfermedad y los cuidados; las dificultades en la adhesión al tratamiento tenían aspectos socioculturales, los cuales podrían ser negociados entre los adolescentes y sus familiares.

DESCRIPTORES: Enfermería; Diabetes mellitus tipo 1; Salud del adolescente.

${ }^{1}$ Enfermeira. Mestranda em Cuidados Clínicos. Universidade Estadual do Ceará. Fortaleza, CE, Brasil.

${ }^{2}$ Enfermeira. Universidade Estadual do Ceará. Fortaleza, CE, Brasil.

${ }^{3}$ Enfermeira. Doutora em Enfermagem. Docente do Programa de Pós-Graduação em Cuidados Clínicos na Universidade Estadual do Ceará. Fortaleza, CE, Brasil. 


\section{- INTRODUÇÃO}

O Diabetes Mellitus tipo1 (DM1) é uma doença crônica, autoimune, que pode acometer diferentes faixas etárias, sendo mais comumente diagnosticada em criança, adolescente e jovem adulto. Esta tipologia corresponde a cerca de 5-10\% dos casos de diabetes. Caracteriza-se pela destruição progressiva e insidiosa das células $\beta$ produtoras de insulina das ilhotas pancreáticas, desencadeando a deficiência absoluta de insulina. Requer cuidados contínuos e educação em saúde para o autocuidado, com vistas a prevenir complicações agudas e reduzir o risco de complicações crônicas ${ }^{(1-2)}$.

A adolescência é marcada pela transição da infância para a vida adulta, permeada pelo desenvolvimento físico e biopsicossocial. Pode se configurar em uma etapa difícil e conflituosa, principalmente pelas particularidades que envolvem a afirmação consigo, com a família, o grupo de amigos e a sociedade. Entretanto, pode ser ainda mais difícil quando vivenciada ante o adoecimento, isto é, mediante convívio com uma doença crônica,como a DM1 $1^{(3)}$.

Ao receber o diagnóstico de DM1, o adolescente se encontra em uma situação conflituosa, pois o adoecimento requer mudanças permanentes no cotidiano. As doenças crônicas exigem do indivíduo adaptação às limitações. Essas mudanças são intensificadas quando setratam de crianças e/ou adolescentes, pois o esperado é que estas vivam situações de saúde, para crescer e se desenvolver dentro dos limites da normalidade ${ }^{(4)}$.

Frente a isso, o apoio e a disposição da família para ajudar o adolescente a aceitar a doença poderão contribuir para o enfrentamento desta. O profissional de saúde, além de acolher o adolescente, deve orientar a família sobre o diabetes e o tratamento. Portanto, o enfermeiro é um dos responsáveis por tal ação(5).

Para tanto, faz-se necessário provocar mais escuta e descobrir maneiras de sensibilizar e disseminar conhecimentos entre adolescentes com DM1, para que possam conceituar experiências e assumir o autocuidado. A atuação do enfermeiro inclui o aconselhamento para uma vida saudável dos adolescentes, buscando diminuição dos agravos, no contexto do viver com DM1 ${ }^{(6)}$.

Nesta perspectiva, para que os adolescentes assumam cuidados e controle do esquema terapêutico, os profissionais devem identificar necessidades e ajudá-los a se sentirem responsáveis e capazes de cuidarem de si. Logo, é fundamental que a equipe cuidadora desenvolva uma educação em saúde participativa, envolvendo o sujeito adoecido e a família, conhecendo a realidade sociocultural e os aspectos clínicos dos usuários assistidos pelo sistema de saúde.

Para desenvolver a pesquisa, formulou-se o questionamento: quais os aspectos socioculturais e clínicos de adolescentes com Diabetes Mellitus tipo 1 que podem subsidiar atividade educativa em grupo?

Dessa forma, objetivou-se descrever os aspectos socioculturais e clínicos de adolescentes com Diabetes Mellitus tipo 1 e relatar atividade educativa em grupo com base nas necessidades deste público-alvo.

\section{MÉTODOS}

Trata-se de estudo exploratório, descritivo, configurado em duas etapas de coleta de dados. A primeira teve predominância de dados quantitativos apreendidos em entrevista semiestruturada aplicada individualmente. Na segunda etapa, foi realizada atividade educativa com os adolescentes participantes da primeira etapa.

A população do estudo foi de 86 adolescentes e a amostra foi por conveniência, composta por 38 adolescentes com DM1, que atenderam aos seguintes critérios de inclusão: com 10 a 19 anos de idade, acompanhados no Centro de Diabetes e Hipertensão (CIDH) com diagnóstico há, no mínimo, seis meses, cujo prontuário encontrava-se disponível no período da pesquisa. Foram excluídos adolescentes que apresentaram intercorrência clínica, como sintomas de hipoglicemia ou hiperglicemia, o que poderia comprometer os informantes e as informações.

As informações foram extraídas por roteiro semiestruturado, contemplando aspectos socioculturais e clínicos, além de uma pergunta aberta sobre os cuidados para manter o controle da glicemia. O período do estudo foi de janeiro a junho de 2015, as interações com os sujeitos e a recolha dos dados ocorreu de abril a 
maio de 2015. Os dados foram digitados e processados no software Statistical Package for the Social Sciences (SPSS), versão 20.0, em que se utilizou estatística descritiva.

Os adolescentes que participaram da primeira etapa foram comunicados pessoalmente e reforçado o convite, por ligação, a participarem da atividade educativa em grupo. Foi combinado com eles e o responsável, como também com a enfermeira do serviço, que apoiou e cedeu o espaço. O primeiro encontro marcado não ocorreu devido ao não comparecimento, no segundo, contou-se com cinco participantes. As comunicações foram registradas com recurso áudio e visual.

No segundo momento, realizou-se atividade educativa em grupo com parte dos adolescentes que, intencionalmente, foram escolhidos, considerando a presença destes no serviço. A atividade educativa elaborada pelas pesquisadoras teve como objetivo desenvolver dinâmicas, diálogos e reflexões sobre o cuidado em relação à alimentação junto ao adolescente com Diabetes Mellitus. As estratégias desenvolvidas foram: convite e acolhimento; roda de conversa sobre alimentação e diabetes; aplicação de dinâmica: alimentos recomendados e não recomendados; discussão e reflexão. Utilizaram-se como recursos materiais: figuras/imagens.

Na interação com os adolescentes, após o acolhimento e explicações da dinâmica, foram estimuladas as conversas sobre as orientações alimentares recebidas durante a consulta com os profissionais; motivações e dificuldades na escolha dos alimentos da dieta. Em seguida, iniciou-se a identificação e classificação das figuras de alimentos recomendados e os não recomendados.

Os participantes elaboraram dois quadros representando esses alimentos; respeito pelos intervalos de três em três horas, aumento da ingesta hídrica e, espontaneamente, discorreu sobre a rotatividade do local de aplicação da insulina, a lavagem das mãos antes de iniciar a aplicação da medicação e os cuidados com os pés. Após o compilado de ideias, com base nos quadros apresentados e nas conversas geradas entre eles, as pesquisadoras estimularam o relato sobre o dia a dia, comentaram o seu cotidiano e as dúvidas geradas foram esclarecidas, mantendo-se linguagem acessível aos adolescentes e ressaltando-se os benefícios de determinados alimentos, lembrando-os de associar a importância da dieta para o melhor controle glicêmico, como também dos medicamentos e da atividade física.

A avaliação foi realizada pela observação da participação dos adolescentes nas atividades, dúvidas e questionamentos gerados por cada participante.

O estudo cumpriu os preceitos éticos da Resolução 466, de 12 de dezembro de 2012, que regulamenta pesquisas envolvendo seres humanos ${ }^{(7)}$. O projeto foi aprovado pelo Comitê de Ética em Pesquisa da instituição promotora da pesquisa, conforme parecer no 181.489, da Universidade Estadual do Ceará. Assim, os procedimentos de proteção dos sujeitos da pesquisa e da instituição foram efetivados, considerando os princípios da bioética ressaltados na referida Resolução. Foi explicado o termo de consentimento aos responsáveis e de assentimento aos adolescentes, esclarecendo a respeito da pesquisa, do anonimato e da autonomia dos direitos garantidos, pois tinham a liberdade de participar ou não.

\section{RESULTADOS}

Os aspectos socioculturais e clínicos dos adolescentes pesquisados com DM1 foram analisados, obtendose os resultados a seguir.

Dentre os 38 adolescentes entrevistados, 20 (52,6\%) eram do sexo masculino, enquanto 18 (47,3\%) do sexo feminino. Em relação à procedência, 21 (55,2\%) residia em Fortaleza, nove $(23,7 \%)$ no interior do Estado e oito $(21 \%)$ na Área Metropolitana. Quanto à escolaridade, $37(97,3 \%)$ se encontravam estudando, $20(52,6 \%)$ cursando o ensino fundamental, $14(36,8 \%)$ o ensino médio e três $(7,9 \%)$ o ensino superior. Ao considerar a faixa etária dos participantes (10-19 anos), constatou-se compatibilidade com o nível de escolaridade, pela variação de idade.

Em relação à renda familiar dos participantes, 20 (52,6\%) sobrevivia com um salário mínimo, seguido de $15(39,5 \%)$ com dois a três salários mínimos e quatro (10,5\%) de quatro a mais salários. As condições de moradia apresentavam-se favoráveis 32 (84,2\%), ou seja, a maioria relatou morar casa própria. Além disso, $17(44,7 \%)$ informaram residir com cinco pessoas ou mais, seguindo de $11(28,9 \%)$ com três pessoas e oito $(21 \%)$ com quatro pessoas. Quanto à convivência familiar, $26(68,4 \%)$ moravam com os pais, retratando a 
situação de dependência e, ao mesmo tempo, de ajuda recebida dos pais.

A participação em grupos sociais obteve percentual de 35 (92,1\%), denotando superação das dificuldades de preconceito e vergonha em convivência social, pois três $(7,9 \%)$ responderam não participar de grupos sociais.

Quanto aos aspectos de saúde demonstrados nos achados clínicos dos adolescentes pesquisados, observou-se que a maioria, $20(52,6 \%)$ apresentou IMC normal $(18,5-24,9)$, seis $(15,8 \%)$ abaixo do peso ideal e dois (5,3\%) acima do peso. A glicemia capilar em jejum, da maior parte dos adolescentes, 27 (71\%) correspondeu ao estado de hiperglicemia (acima de $100 \mathrm{mg} / \mathrm{dl}$ ) e oito $(21 \%)$ apresentaram glicemia normal em jejum. Sobre o tempo em relação ao diagnóstico inicial até o momento, 20(52,6\%) dos participantes afirmaram ter mais de quatro anos, $14(36,8 \%)$ de dois a quatro anos e quatro $(10,5 \%)$ aproximadamente seis meses.

Os principais sintomas que apresentaram no início do diagnóstico foram: a presença de poliúria, polifagia, polidipsia, perda de peso, hiper e hipoglicemia 16 (42,1\%); poliúria, polidpsia e polifagia 11 (28,9\%) e perda de peso, sinais de hiper e hipoglicemia nove $(23,7 \%)$.

Em relação à terapia medicamentosa, $18(47,4 \%)$ faziam uso da insulina Neutral Protamine Hagedorn (NPH) e da insulina regular, enquanto $16(42,1 \%)$ de análogos de ação rápida e três $(7,9 \%)$ combinação dos análogos com NPH e/ou regular. $\mathrm{Na}$ insulinoterapia 37 (97,3\%) dos adolescentes realizavam esse procedimento, sete $(18,4 \%)$ referiram que a aplicação era realizada pela mãe ou por outros familiares.

A respeito da atividade física, 30 (78,9\%) responderam que praticavam rotineiramente e oito (21\%) dos adolescentes não realizavam exercício físico. Dentre os que desempenhavam atividade, as mais recorrentes foram os esportes coletivos 16 (42,1\%), como futebol, basquete, vôlei e a própria educação física oferecida nas escolas, semanalmente. Cerca de cinco $(13,1 \%)$ dos entrevistados responderam praticar mais de uma modalidade esportiva.

Quanto ao autocuidado e às atitudes para manter o controle da doença, os adolescentes responderam praticá-las diariamente, como condição inerente à vida. As medidas de autocuidado que se destacaram foram controle alimentar, prática de exercício físico, uso correto das medicações, acompanhamento regular com o profissional de saúde até o cuidado com os pés e a pele. Constatou-se que $17(44,7 \%)$ praticavam quatro ou mais dessas medidas, focando-se em medidas de controle da glicemia, como alvo principal de cuidados.

Na segunda etapa, após análise do material coletado na entrevista, extraiu-se como problemas vivenciados pelos adolescentes, o cumprimento da dieta, ou seja, as dificuldades enfrentadas, principalmente, nos ambientes sociais. Deste modo, efetivou-se atividade educativa no grupo, tendo como foco o cuidado na alimentação dos adolescentes com DM1. Foram convidados os participantes da primeira etapa, mas apenas cinco compareceram, demonstraram interesse e participaram ativamente da discussão referente à alimentação.

\section{- DISCUSSÃO}

Os dados encontrados no estudo mostram que as condições de vida e renda dos sujeitos pesquisados eram desfavoráveis à obtenção de saúde, pois a maioria sobrevivia com um salário mínimo, dentre outros aspectos associados. Isto se agrava quando o sujeito é acometido por doença crônica que depende de assistência especializada, tecnologias e cuidados contínuos, portanto, acesso a recursos e bens de consumo, incluindo alimentação diferenciada.

Ratificando com a literatura, o Diabetes tipo 1 é uma doença crônica, grave, com efeito prejudicial sobre a qualidade de vida, principalmente em crianças e adolescentes, atingindo toda a família ${ }^{(8)}$. Os adolescentes têm história de vida, renda e grau de escolaridade destes e dos pais podem influenciar no controle glicêmico ${ }^{(9)}$.

O contexto no qual o adolescente está inserido, muitas vezes, dificulta a adequação ao tratamento do diabetes. Dentre os elementos a serem considerados na adesão/adequação a esse processo, citam-se: as metas e os objetivos de autocontrole, o ambiente, os marcos do desenvolvimento, a progressão cognitiva, dentre outros ${ }^{(10)}$. 
O tratamento do Diabetes tipo 1 é uma complexa terapia medicamentosa e não medicamentosa, que ocasiona impacto emocional e mental, fazendo com que os adolescentes experimentem emoções negativas, incluindo raiva, preocupação, constrangimento, ansiedade em relação ao cotidiano e às possíveis ocorrências ${ }^{(8,11)}$.

A maioria dos adolescentes desta pesquisa relatou que o tratamento medicamentoso, na maior parte, seguia o esquema terapêutico na conjunção das insulinas NPH e regular, pois estas drogas respondem ao tratamento, diminuindo a ocorrência de complicações crônicas, apesar de existirem outras terapêuticas mais eficazes, em termos gerais, mas que são mais onerosas ${ }^{(2,12)}$.

As práticas citadas pelos adolescentes no manejo da doença envolvendo o autocuidado evidenciaram que o tratamento integrava medidas medicametosas e não medicamentosas. O automanejo é complexo, exige monitorização contínua dos níveis de insulina no sangue, readequação alimentar, doses diárias de insulina, prática de atividades físicas, o que gera estresse no adolescente, na família e díficil controle metabólico ${ }^{(13-14)}$.

Em face desse contexto, os pais devem oferecer apoio social, emocional, equilíbrio e independência ao adolescente, subsidiando de forma positiva o tratamento, não ultrapassando limites, dispondo ao adolescente ser o autor do autocuidado e autocontrole, pois são ferramentas importantes. Vigilância constante pode causar sofrimento, mas aprender a enfrentar as situações o tornará um adulto sócio, espiritual, emocional, mentalmente saudável ${ }^{(15-16)}$. Visualizou-se, na pesquisa, independência do adolescente no manejo com a insulina, pois a maioria relatou que fazia autoaplicação e não deixará transparecer maiores dificuldades em lidar com este procedimento.

Por outro lado, a maioria relatou comer na escola alimentos não adequados como salgados e refrigerantes, uma pequena parcela referiu comer frutas, mas que traziam de casa, porém sabe-se da dificuldade que as famílias apresentam no acesso a uma alimentação a base de frutas, verduras ou outro nutriente de baixa caloria, pois o custo é maior em relação aos alimentos mais calóricos. Portanto, as escolas devem apresentar recursos físicos, materiais e humanos para contemplar as condutas adequadas no manejo com o DM1. Para tanto, é preciso capacitação de professores para dominar o conhecimento específico sobre a diabetes, detendo conhecimentos gerais e específicos, principalmente no que refere à saúde escolar ${ }^{(17)}$.

Destaca-se a necessidade de intensificar o acompanhamento nutricional de pacientes com diabetes, obtendo-se maior adesão às restrições alimentares e melhor controle glicêmico. Para isso, é necessário ofertar diversidade de cardápios e planejar junto ao paciente melhor oferta de alimentos, favorecendo o controle metabólico ${ }^{(9)}$.

A regulação da glicemia sanguínea no DM1 é uma das maiores dificuldades, principalmente com os adolescentes, devido às mudanças no desenvolvimento físico e mental, com mais comprometimento no controle metabólico.

No estudo, identificou-se que a maioria apresentava hiperglicemia em jejum, assim inferindo alimentação inadequada, principalmente no período noturno. A despeito do incentivo à autonomia destes sujeitos para assumirem o autocuidado, os pais de crianças e adolescentes com diabetes têm grande influência na monitorização e no controle da glicemia, além de acompanhamento do autocuidado, como adaptação ao estilo de vida saudável, dieta, prática de atividades físicas e administrações das injeções ${ }^{(8)}$.

Os achados da pesquisa ainda mostram que a maioria adotava a prática da atividade física, no propósito de conseguir melhor resposta metabólica e controlar os níveis glicêmicos. O estilo de vida sedentário tem se tornado um dos fatores que contribuem para as dificuldades do controle metabólico da glicose no sangue em pacientes com DM1. Destarte, as atividades físicas devem estar inclusas nas orientações clínicas, pois a ausência destas tem associação direta com o baixo controle metabólico ${ }^{(18-19)}$.

São necessárias abordagens criativas e participativas. Na pesquisa, foi possível desenvolver estratégias participativas, favorecendo interação entre os adolescentes, que, por meio da atividade, puderam expor conhecimentos e, de forma dinâmica, apreender sobre doença e tratamento.

Os grupos educativos auxiliam a desenvolver capacidades e habildades que estimulam a participação e o autogerenciamento da doença. Contudo, para mudar estilo de vida e atitudes perante o tratamento, urge agregar outras estratégias, como sites, a própria consulta com a equipe de saúde, para o real impacto no tratamento e no controle glicêmico, além de permitir que indivíduos interajam com outras pessoas com semelhante condição de saúde, compartilhando experiências, podendo aprender mais e associar melhor as 
informações ${ }^{(8,16,20)}$.

Assim, elaborou-se atividade educativa aplicada junto aos adolescentes, momento em que ocorreu troca de experiências e dinamismo para os adolescentes.

Os profissionais de saúde poderiam estimular a participação dos adolescentes em atividades educativas, convidando-os e, através de dinâmicas interativas, promoverem discussões em grupos com os participantes, permitindo que estes tenham voz ativa no processo de comunicação ${ }^{(16)}$. Os resultados sugeriram que as orientações dos profissionais estão sendo compreendidas pelos adolescentes, pois a maioria conhecia as medidas terapêuticas de autocuidado.

Diante disso, é relevante a realização da atividade em grupo mediada pelo enfermeiro e por ações educativas em saúde, cujo profissional torna-se ouvinte das necessidades biopsicosociais do usuário, sugerindo estratégias para adesão ao autocuidado ${ }^{(8,21)}$.

Ao conhecer o público de adolescentes diabéticos, foi possível identificar que a maioria compreendia o tratamento, mas apresentava dificuldades de executá-lo no cotidiano, trazendo complicações, como o não controle alimentar e hiperglicemia em jejum. Assim, com a intervenção educativa e a dinâmica em grupo, foi possível dispor de maior interação e troca entre os participantes, no manejo de hábitos saudáveis, enfrentamento e adaptação à situação de diabético e tratamento da doença, partilha que traz mudanças positivas acerca de mudanças nos hábitos.

\section{CONSIDERAÇÕES FINAIS}

Descreveram-se os aspectos socioculturais e clínicos dos adolescentes com Diabetes Mellitus tipo 1 e relatou-sea atividade educativa em grupo, com base nas necessidades do público-alvo, permitindo conhecer as dificuldades enfrentadas pelos adolescentes participantes, na convivência com o diabetes e o cumprimento das recomendações alimentares.

O desenvolvimento da atividade educativa com produção de material elaborado pelos adolescentes estimulou debate e, consequentemente, problematização de questões do cotidiano sobre a escolha e a quantidade dos alimentos; associação da dieta com o controle glicêmico e prevenção de complicações. Os adolescentes afirmaram não gostar de consumir os alimentos recomendados prescritos nas dietas e consumem no café da manhã pão com manteiga e leite. Outro ponto ressaltado foi a dificuldade para manter a orientação alimentar na escola, pois convivem com os colegas que consumem salgados e refrigerantes. Dois adolescentes relataram levar frutas para o lanche, um afirmou lanchar papa de farinha de trigo fornecida pela escola e outro, rotineiramente, levar biscoitos para o lanche.

A atividade educativa realizada permitiu ao adolescentecom DM1 conhecer e entender aspectos importantes da dieta, a fim de facilitar a adoção de uma alimentação saudável em sua prática. Isto foi possível a partir da identificação das necessidades, mediante aproximação com os adolescentes. Notaram-se dificuldades dos sujeitos em relação à manutenção da alimentação adequada, devido a problemas sociais e econômicos, como previstos nas recomendações profissionais constantes, também no plano de ensino elaborado pelos pesquisadores.

A aproximação com este público trouxe percepção acerca das características gerais e individuais. Assim, foram percebidas também necessidades educativas que favoreçam apreensão de conhecimentos sobre o adoecimento e a importância do tratamento e autocuidado, a fim de prevenir possíveis complicações e proporcionar melhor bem-estar. Apesar de o adolescente deter conhecimentos acerca do tratamento e das complicações da doença, muitos não adotavam o tratamento, evidenciando-se a necessária intervenção educativa.

Nesse ensaio educativo, obteve-se troca de experiências com os adolescentes, que expressaram, além dos conhecimentos, curiosidades, dúvidas acerca do tratamento e do cuidado. Configurou-se, portanto, como ação dinâmica, com estratégia criativa e participação dos sujeitos, permitindo o diálogo e a reflexão da situação vivenciada por cada um, no controle alimentar.

A partir deste estudo, pode-se inferir o desafio profissional de desenvolver atividades educativas, com participação ativa dos adolescentes e familiares, e constante atuação da equipe de saúde. Embora tenha sido 
aberto um canal de comunicação mais efetivo para discorrer sobre a subjetividade de cinco adolescentes, as limitações estão postas em virtude das observações apreendidas não alcançarem outros sujeitos e contextos diferenciados, ampliando as discussões.

Faz-se necessário, portanto, estudos referentes ao modo de efetivar tais intervenções na enfermagem, frente às necessidades e aos cuidados com esses adolescentes, aprimorando as ações nos serviços de saúde, proporcionando eficácia no tratamento, reduzindo danos à saúde e melhorando a qualidade de vida destes.

\section{AGRADECIMENTOS}

Agradecemos ao Conselho Nacional de Desenvolvimento Científico e Tecnológico (CNPq) que financiou esta pesquisa № 485359/2012-0 e ofereceu subsídios para o seu desenvolvimento.

\section{- REFERÊNCIAS}

1. Gomes MB, Cobas R. Diabetes mellitus. In: Grossi SAA, Pascali PM, organizadoras. Cuidados de enfermagem em Diabetes Mellitus: manual de enfermagem. São Paulo: Departamento de Enfermagem da Sociedade Brasileira de Diabetes; 2009. p. 6-17.

2. Sociedade Brasileira de Diabetes (SBD). Diretrizes da Sociedade Brasileira de Diabetes: 2014-2015. São Paulo:AC Farmacêutica; 2015.

3. Ferreira LE, Zanatta EA, Brumm MLB, Nothaft SCS, Motta MGC. Diabetes Mellitus sob a ótica do adolescente. CogitareEnferm. 2013; 18(1): 71-7.

4. Leal DT, Fialho FA, Dias IMAV, do Nascimento L, Arruda WC. A vivência dos familiares de crianças e adolescentes portadores de diabetes mellitus tipo 1. Rev. Eletr. Enf. 2012; 14(1): 189-96.

5. de Oliveira APL, Sarmento SS, Mistura C, Jacobi CS, Girardon-Perlini NMO, Carvalho e Lira MOS,et al. Experiência de familiares no cuidado a adolescentes com diabetes mellitus tipo 1. RevEnferm UFSM. 2013; 3(1): 133-43.

6. Araújo YB, Reichert APS, de Vasconcelos MGL, Collet N. Fragilidade da rede social de famílias de crianças com doença crônica. Rev. bras. enferm. 2013;66(5):675-81.

7. Ministério da Saúde (BR). Conselho Nacional de Saúde. Diretrizes e normas regulamentadoras de pesquisa envolvendo seres humanos. Resolução n. 466, de 12 de dezembro de 2012. Brasília: Ministério da Saúde; 2012.

8. Konradsdottir E, Svavarsdottir EK. How effective is a short-term educational and support intervention for families of an adolescent with type 1 diabetes?] SpecPediatrNurs.2011; 16(4): 295-304.

9. Queiroz KC, Silva IN, Alfenas RCG. Associação entre fatores nutricionais e o controle glicêmico de crianças e adolescentes com diabetes melito tipo 1. Arq Bras EndocrinolMetab. 2010; 54(3): 319-25.

10. Cooper H, Spencer J, Lancaster GA, Titman A, Johnson M, Wheeler SL, et al. Development and psychometric testing of the online Adolescent Diabetes Needs Assessment Tool (ADNAT). J AdvNurs. 2014; 70(2): 454-68.

11. Kime N, Mckenna J, Webster L. Young people's participation in the development of a self-care intervention-a multisite formative research study. Health Educ Res. 2013;28(3):552-62.

12. Marques RMB, Fornés NS, StringhiniMLF.Fatores socioeconômicos, demográficos, nutricionais e de atividade física no controle glicêmico de adolescentes portadores de diabetes melito tipo 1. ArqBrasEndocrinolMetab. 2011; 55(3): 194202.

13. Whitremore R, Jaser SS, Faulkner MS, Murphy K, Delamater A, Grey M,et al. Type 1 diabetes eHealthpsychoeducation: youth recruitment, participation and satisfaction.J Med Internet Res.2013; 15(1): e15.

14. Froisland DH, Arsand E, Skarderud F. Improving diabetes care for young people with type 1 diabetes through visual learning on mobile phones: mixed-methods study.J Med Internet Res. 2012; 14(4): e111. 
15. Barreto MS, da Silva AM, Nortean ECM, Marcon SS. Conviver com diabetes mellitus sob a ótica de adolescentes e jovens e suas mães. Rev. pesqui. cuid. fundam. 2012; 4(4): 3080-93.

16. Noordman J, van Dulmen S. Shared Medical Appointments marginally enhance interaction between patients: An observational study on children and adolescents with type 1 diabetes. PatientEducCouns. 2013; 92(3): 418-25.

17. Simões ALA, Stacciarin TSG, Poggetto MTD, Maruxo HB, Soares HM, Simões ACA.Conhecimento dos professores sobre o manejo da criança com diabetes mellitus.TextoContextoEnferm.2010; 19(4): 651-7.

18. Benevento D, Bizarri C, Pitocco D, Crinò A, Moretti C, Spera S, et al.Computer use, free time activities and metabolic control in patients with type 1 diabetes. Diabetes Res ClinPract.2010; 88(3): e32-4.

19. Miculis CP, Mascarenhas LP, BoguszewskiMCS,deCampos W. Physical activity in children with type 1 diabetes. J. Pediatr. 2010; 86(4): 271-8.

20. Christie D, Thompson R, Sawtell M, Allen E, Cairns J, Smith F, et al. Structured intensive education maximising engagement, motivation and long-term change for children and young people with diabetes a cluster randomized controlled trial with integral process and economic evoluation - the CASCADEstudy. Health Technol Assess.2014; 18(20): $1-202$.

21. Pennafort VPS, Silva ANS, Queiroz MVO. Percepções de enfermeiras acerca da prática educativa no cuidado hospitalar a crianças com diabetes.Rev. Gaúcha Enferm. 2014; 35(3): 130-6. 